\title{
Perioperative Care of Children with Acute Exogenous Lipoid Pneumonia Submitted to BAL/FB
}

\author{
Wen-Chun Lin ${ }^{1} *$ \\ Yi Qin ${ }^{1, *}$ \\ Xiao Chun ${ }^{2}$ \\ Ru-Lin Huang' \\ Rong-Shan Chen' \\ Dongwei Zhang'
}

'Department of Respiratory, Guangzhou Women and Children's Medical Center, Guangzhou Medical University, Guangzhou, Guangdong, People's Republic of China; ${ }^{2}$ Pediatric Intensive Care Unit, Guangzhou Women and Children's Medical Center, Guangzhou Medical University, Guangzhou, People's Republic of China

*These authors contributed equally to this work
Correspondence: Dongwei Zhang Department of Respiratory, Guangzhou Women and Children's Medical Center, Guangzhou Medical University,

Guangzhou, 510120, People's Republic of China

Tel +862038076135

Fax +862038076626

Email zhangdw137@I26.com
Objective: The present study evaluates the psychosocial care and the bronchoalveolar lavage (BAL)/fiberoptic bronchoscopy $(\mathrm{FB})$ procedure in children with pediatric acute exogenous lipoid pneumonia (ELP) and summarizes the critical points of nursing.

Methods: Data on the psychosocial factors of the patients and clinical information were collected. Participants comprised 41 children within three years of age.

Results: All the children were cooperative with the BAL/FB procedure. The children's pain scores were between 4-6, and the psychological conditions of the children and caregivers were nervous/anxious upon admission. After the medical staff's psychological care and health education, the children's postoperative pain scores were reduced to $0-3$, and the psychological state of the caregivers was positive.

Conclusion: Psychological care can alleviate families' adverse emotions and promote treatment cooperation and recovery from the acute ELP.

Keywords: children, acute exogenous lipoid pneumonia, psychological care, bronchoscopy, bronchoalveolar lavage

\section{Introduction}

Acute exogenous lipoid pneumonia (ELP) exposes children and their parents to extreme stressors. ${ }^{1-4}$ It is caused by inhaling grease substance, such as paraffin oil, cod liver oil, gasoline, etc., cause pulmonary inflammation and local tissue fibrosis. $^{1-4}$ Bronchoalveolar lavage (BAL) with pediatric flexible fiberoptic bronchoscopy $(\mathrm{FB})$ is used in pediatric acute ELP. ${ }^{5,6}$ Etiologic agents of ELP can vary depending on differences in the affected countries' cultures and lifestyles. ${ }^{7}$ Some customs and habits, such as using lamp oil/sesame oil, are the risk factors in China. $^{2}$

Traditional treatment methods ignore the importance of psychological care for patients. Psychological health is an important predictor for coping with difficult events and adjusting to the psychological and social demands placed on children diagnosed with ELP. Parental stress is affected by children's behavior. ${ }^{8-14}$ Pediatric nurses play an important role in psychological care. First, the efficacy of psychological care in acute ELP is evident in our presented cases. Although most primary caregivers and children had fear, anxiety, and other adverse emotions in the early stage of admission, these can be alleviated through good psychological care. Therefore, we need to assess children's pain levels, especially younger children 
unable to express themselves. Thus, the current focus on acute ELP treatment should not be limited to disease control but should extend to psychological care. In the course of the $\mathrm{BAL} / \mathrm{FB}$ procedure, careful nursing and psychosocial care can improve clinical symptoms and signs and improve the prognosis of pediatric acute ELP patients. This study aims to evaluate psychosocial care and the $\mathrm{BAL} / \mathrm{FB}$ procedure in pediatric acute ELP children and summarize nursing's key points.

The present study evaluates the psychosocial care and BAL/FB procedure in children with pediatric acute exogenous lipoid pneumonia (ELP) and summarizes the critical points of nursing. Hypothesis is that psychological care can alleviate families' adverse emotions and promote treatment cooperation and recovery from the acute ELP.

\section{Method}

\section{Design and Sample}

The study group was 41 pediatric patients. Diagnostic bronchoscopy and BALs for treatment were performed immediately after admission if there were no contraindications. In the first three years, the authors performed BAL weekly. BALs were performed twice in the first week, and subsequent BALs were performed weekly if necessary. Thus, the interval between BALs was shorter than before, and the volume of BAL increased by $50 \%$ in the past two years. Inhaled corticosteroid was given before and after the BAL/ FB procedure to reduce complications such as throat edema. We evaluated the safety and efficacy of the BAL/FB procedure and psychosocial care in children with acute ELP. This study was conformed to the Declaration of Helsinki and was approved by the Ethics Committee of our hospital. Informed consent was signed by all participants' guardians.

\section{Data Collection}

Demographic data, categories of oil-ingested BAL findings, the social and psychological condition of the children and families, and outcomes were collected. Psychosocial problems were assessed routinely to initiate targeted psychological care for every child and family, during which the families' mood and therapeutic compliance were recorded.

\section{Nursing}

The cases' preoperative examinations such as routine examination of cardiopulmonary function, routine blood tests, and clotting time were evaluated. The nurses also assessed the parents' previous experience and current expectations for bronchoscopy. Parents were encouraged to express their doubts honestly and explained and conveyed the information positively. Before the bronchoscopy operation, the nurses described the methods, effects, and possible complications of bronchoscopy to the parents in detail and plain language, emphasizing the development and prognosis of the disease, the necessity for administering the $\mathrm{BAL} / \mathrm{FB}$ procedure, and the need for positive cooperation with the medical staff to promote the improvement of the condition.

\section{Psychological Care}

A video of the operation was shown before the procedure so that the parents and/or children could understand the process and deepen their understanding of BAL. A cartoon surgery simulation video was available for children. Toys, video games, painting, playing, and music were used to relieve the pre- and postoperative anxiety and fear of children of all ages. ${ }^{7}$ In addition, we should closely assess the pain level of the perioperative period in children using the FLACC scale. ${ }^{8}$

Understanding children's family background, including their primary caregivers' education level, family environment, and economic situation, will affect the way nurses express health education for them and their understanding and decision-making regarding surgery, thus affecting their emotional response. The nurse should also observe family relationships during admission evaluations and comprehensively evaluate their psychological state, especially before the $\mathrm{BAL} / \mathrm{FB}$ procedure. According to the characteristics of the children and their families, individualized nursing care was provided, considering psychology, physiology, family, and social adaptability. For the primary caregivers who use different dialects, such as Cantonese, medical staff who understand such dialects are necessary to understand the content of health education better. We should also choose appropriate language to educate primary caregivers, considering their different educational levels. Nurses encourage the family to make medical decisions and develop preventive and healthy activities together. Psychological care is based on empathetic and supportive communication so that parents can communicate with other family members or parents of children with the same disease. Psychological care does not happen just once but lasts throughout the whole hospitalization process.

Nurses should build an environment similar to the family atmosphere for the child, adjust the humidity and 
ventilation regularly in the ward, establish a good relationship with them, and pay attention when communicating with the children to understand and meet their psychological needs. The treatments and medical procedures should be performed within a concentrated period to reduce children's adverse stimulation.

\section{Preoperative Preparation}

The cases need to be sedated for the $\mathrm{BAL} / \mathrm{FB}$ procedure, but the sedation of children poses a certain risk of aspiration. Preoperative assessment is necessary to ensure safety. For the cases that undergo multiple BALs, inhaled corticosteroid was given before and after the BAL/FB procedure to reduce throat edema.

\section{Operative Nursing Procedures}

Embalming principles must be followed throughout the $\mathrm{BAL} / \mathrm{FB}$ operation. BAL is usually performed in the most severely affected areas using sterile saline (radiological and/ or endoscopic) heated to body temperature $\left(37^{\circ} \mathrm{C}\right)$. During the first three years, the ratio of BAL volume to body weight was $3-5 \mathrm{~mL} / \mathrm{kg}$, and each leaf was divided into $3-5$ equal parts. With the accumulation of experience, the authors increased the ratio of ball volume to body weight to 5$8 \mathrm{~mL} / \mathrm{kg}$ in the past two years. Using a pressure of 6.65 $13.3 \mathrm{kPa}(50-100 \mathrm{~mm} \mathrm{Hg})$, the liquid is recovered by pumping into the suction trap. In general, a BAL is acceptable if $>$ is restored to $40 \%$. During BAL/FB surgery, the airway should be kept clear and unimpeded, and oral and nasal secretions should be removed promptly. Nurses closely monitor the children's vital signs and expressions. If oxygen saturation is found to be less than $80 \%$, surgery is stopped immediately and oxygen flow is increased to ameliorate hypoxia. Continue surgery after oxygen saturation exceeds $95 \%$ to ensure safety. Patient monitoring includes continuous evaluation of heart rate, respiratory function, noninvasive blood pressure, color, and airway position. Supplementary oxygen was used during the procedure until the oxygenation of the room air was recorded. The frequency of BAL depends on radiological, endoscopic, and clinical conditions, and children receiving prolonged sedation should be given extended observation.

\section{Postoperative Care}

Postoperative rehabilitation takes place in post-anesthesia care units equipped with emergency interventions. During recovery, the patient's oxygen saturation and percutaneous oxygen saturation were monitored by electrocardiogram. All patients were fasted and dehydrated for about two hours postoperatively to avoid reflux and aspiration. A transient aggravation of cough, hoarseness, throat discomfort, low fever, and bloody sputum are normal conditions after BAL/ FB surgery and do not require symptomatic treatment and usually resolve after one to two days. If the symptoms are more severe, prompt symptomatic treatment is needed. Nurses need to dynamically observe the consciousness, blood circulation, breathing and so on during the examination, surgery and rehabilitation of children, and timely detect complications in the process of postoperative care.

Although the overall prognosis of acute ELP is good, in some cases, the condition may still be aggravated in the course of treatment, such as increased body temperature, rapid breathing, failure of oxygen intake to improve hypoxia, and pulmonary hemorrhage. The nursing staff should cooperate with the doctor and communicate with the parents in time, explain the outcome again patiently, and let the parents make complete mental preparation for the condition change in such cases.

\section{Health Education and Discharge Guidance}

Each parent was issued an out-of-hospital instruction manual after discharge. The nursing staff worked out a daily care plan with the family members, answered any questions, addressed any problems encountered by the family members in the care process, and modified the care plan according to the children's recovery. As the absorption of lung exudation is relatively slow for acute ELP cases, it is essential to inform parents that they must accompany children to regular outpatient follow-up and follow the doctor's advice.

Most children had mineral oil misuse history because the mineral oil was put in a bottle and was mistakenly drunk by the unattended child. Therefore, it is vital to give parents health education to avoid placing unsafe substances in bottles and storing them in a safe place.

\section{Results}

There were 41 children aged 4 to 53 months, the median age of onset was 20 months, $85.4 \%$ were within 3 years of age (35/41), and $51.2 \%$ were 1 to 2 years old (21/41). All patients underwent bronchoscopy with BAL; 17 cases underwent it once, and 24 cases had to have it more than two times (some were performed in the day-care unit after discharge). In the past two years, the lengths of hospital stay were shorter than the first three years, with a shorter interval between BALs and an increased volume of BAL. Due to the use of inhaled corticosteroids before and after the BAL/FB procedure, complications such as throat edema were not 
observed in the children who underwent multiple BALs in the past two years. All children were discharged from the hospital after the treatment. Nine patients were lost to follow-up, and the symptoms of the remaining children improved within one week to one month (Table 1).

Although most families had negative emotions such as nervous anxiety and fear in the early stage of hospitalization, the negative emotions were relieved with careful psychological counseling. All the children and their families cooperated with the $\mathrm{BAL} / \mathrm{FB}$ procedure and received other treatments positively without any negativity. The children's pain scores were primarily between 46 , and the psychological condition of children and caregivers was nervous/anxious on admission. After the medical staff provided psychological care and health education, the postoperative pain scores were mainly reduced to $0-3$. In this study, only one child came from a welfare institution, and the rest had their parents as primary caregivers. The social and psychological conditions of the children and families are shown in Table 2.

\section{Discussion}

We believe this is the first study to present an extensive series of cases of pediatric acute ELP in nursing care. Our patients lacked such risk factors as swallowing dysfunction or neurological disorders. ${ }^{9,10}$ All subjects were cured with prompt discontinuation of the offending agent, treating any complicating infections, bronchoscopy with multiple BALs, supportive care, and without any complications such as pulmonary fibrosis. In the first three years, BAL was performed with a volume of $3-5 \mathrm{~mL} / \mathrm{kg}$ according to body weight divided into 3-5 parts for each lobe, which was similar to Selma's experience. ${ }^{5}$ However, in the past two years in our cases, the lengths of hospital stay were shorter than the first three years, with a shorter interval between BALs and increased volume of BAL. In addition, complications such as throat edema and complications from the use of inhaled corticosteroids, such as stomatitis and secondary infection before and after the BAL/FB procedure, were not observed in children who underwent multiple BALs. Thus, the BAL/FB procedure is safe and effective with careful preoperative preparation and intraoperative and postoperative nursing care.

In this research, most children were between one and two years old, so their cognitive ability is limited, and they are full of curiosity. Many families commonly use beverage bottles to store oil and place them in the open. This is the most typical cause of oil misuse in children in our study, suggesting that family-prevention cannot be ignored. Health care workers should strongly promote health education. Thus, health education for families to prevent similar incidents from recurring is an integral part of our nursing work.

Table I The Demographic Data and Clinical Characteristics of the Children

\begin{tabular}{|c|c|c|c|c|}
\hline & & Frequency(n) & Percentage (\%) & $\boldsymbol{P}$ \\
\hline \multirow[t]{2}{*}{ Sex } & Male & 27 & 65.85 & \\
\hline & Female & 14 & 34.15 & \\
\hline \multirow[t]{4}{*}{ Age(month) } & $0-12$ & 3 & 7.32 & \\
\hline & $13-24$ & 21 & 51.22 & \\
\hline & $25-36$ & II & 26.83 & \\
\hline & $>36$ & 6 & 14.63 & \\
\hline \multirow[t]{4}{*}{ Type of lipid inhaled } & Mineral oil & 19 & 46.34 & \\
\hline & Plant-based oil & 14 & 34.15 & \\
\hline & Animal-based oil & 5 & 12.20 & \\
\hline & Unclear & 3 & 7.32 & \\
\hline \multirow[t]{3}{*}{ Pain score on admission } & $0-3$ & 16 & 39.02 & \multirow[t]{6}{*}{$<0.0001$} \\
\hline & $4-7$ & 24 & 58.54 & \\
\hline & $>7$ & I & 2.44 & \\
\hline \multirow[t]{4}{*}{ Postoperative pain score } & $0-3$ & 36 & 87.80 & \\
\hline & $4-7$ & 5 & 12.20 & \\
\hline & $>7$ & 0 & 0 & \\
\hline & Total & 41 & 100.0 & \\
\hline
\end{tabular}


Table 2 Social and Psychological Condition of the Children and Family

\begin{tabular}{|c|c|c|c|}
\hline & & Frequency(n) & Percentage (\%) \\
\hline \multirow[t]{3}{*}{ Childbearing history } & First child & 15 & 36.59 \\
\hline & Second child or above & 25 & 60.98 \\
\hline & Unknown & I & 2.44 \\
\hline \multirow[t]{2}{*}{ Previous hospitalization history for respiratory diseases } & Yes & 10 & 24.39 \\
\hline & None & 31 & 75.61 \\
\hline \multirow[t]{3}{*}{ The educational level of the primary caregiver } & Primary education and below & 3 & 7.32 \\
\hline & Secondary school education & 21 & 51.22 \\
\hline & College degree or above & 17 & 41.46 \\
\hline \multirow[t]{3}{*}{ Source of medical expenses } & Self-paying & 17 & 41.46 \\
\hline & Medical insurance-paying & 22 & 53.66 \\
\hline & Unknown & 2 & 4.88 \\
\hline \multirow[t]{2}{*}{ Concerns about hospital cost } & Yes & 12 & 29.27 \\
\hline & None & 29 & 70.73 \\
\hline \multirow[t]{2}{*}{ Primary caregiver's language } & Mandarin & 24 & 58.54 \\
\hline & Cantonese (the local dialect) & 17 & 41.46 \\
\hline \multirow[t]{2}{*}{ Primary caregiver's understanding of the disease or the procedure } & Not knowing these & 28 & 68.29 \\
\hline & Know only part of these & 13 & 31.71 \\
\hline \multirow[t]{3}{*}{ Primary caregiver's psychological reaction on admission } & Relaxed & 5 & 12.20 \\
\hline & Concerned & 19 & 46.34 \\
\hline & Nervous/anxious & 17 & 41.46 \\
\hline \multirow[t]{3}{*}{ The child's psychological reaction on admission } & Relaxed & 19 & 46.34 \\
\hline & Nervous/anxious/crying & 22 & 53.66 \\
\hline & Total & $4 I$ & 100.0 \\
\hline
\end{tabular}

In our study, most children at admission were nervous, anxious, or crying, and their pain score was usually 4-6. These children's mental development is still immature, and their tolerance of pain and degree of treatment cooperation is low. For the parents, the sudden onset and rapid progression of acute ELP may lead to emotions such as fear, anxiety, and despair due to a lack of medical knowledge about the disease and understanding of the BAL/FB procedures. In our research, most primary caregivers knew nothing about the disease and the procedure, while a few had partial knowledge.

It is crucial to strengthen psychological guidance and health education. Psychological counseling for children and their parents is essential. Additionally, ELP often requires multiple BALs, which, as an invasive operation, leads some family members to accept the treatment with difficulty. ${ }^{15}$ In our study, 24 (58.53\%) cases underwent bronchoscopy with BAL more than twice. Improving the parents' disease cognition level and treatment confidence can increase treatment compliance.
With the psychological care demonstrated in detail above, all the caregivers gave cooperation positively and smoothly without any depressive symptoms or negative emotions for the procedure. All the cases had a favorable outcome without postoperative complications. The postoperative pain score for the children was relatively low, between $0-3$. Children with acute ELP that received support from family and healthcare providers had a better psychological adjustment in our observations, consistent with an earlier study. ${ }^{15}$

\section{Relevance to Clinical Practice}

In this study, nursing personnel applied their practices, psychological care for the patients and their families, preoperative preparation, operative nursing procedures, and postoperative care. Nurses summarized that good nursing is of great significance in improving the therapeutic effect of the $\mathrm{BAL} / \mathrm{FB}$ procedure in the course of treatment. Psychological care can alleviate families' adverse emotions and promote the degree of treatment cooperation and recovery from the acute ELP. 


\section{Conclusion}

Good nursing is of great significance in improving the therapeutic effect of the BAL/FB procedure in the course of treatment. If the nursing staff performs nursing work with a high sense of responsibility, sensitive observation, and quick reactions, this contributes to the timely detection of condition change and helps prevent complications, while a meticulous, high-quality, and warm nursing staff can ease the negative emotions of the children and family members and encourage their active cooperation with treatment. Strengthening psychological care helps the family to take better care of the children and follow the doctor's advice and the children to actively cooperate with the treatment, thus improving the treatment's effect. The nursing care before, during, and after the BAL/FB procedure should be strengthened. Appropriate nursing measures should be put in place at each step to prevent the occurrence of accidents, ensure the smooth progress of the BAL/FB procedure, and promote the recovery of the children.

\section{Ethics Approval and Consent to Participate}

This study was conformed to the declaration of Helsinki and approval by the Ethics Committee of Guangzhou Women and Children's Medical Center. Written informed consent was signed by all participants' parents.

\section{Funding}

The authors received no financial support for the research, authorship, and/or publication of this article.

\section{Disclosure}

The authors declared no potential conflicts of interest.

\section{References}

1. Hadda V, Khilnani GC. Lipoid pneumonia: an overview. Expert Rev Respir Med. 2010;4(6):799-807. doi:10.1586/ers.10.74
2. Lu G, Xie Y, Huang L, et al. Study of acute exogenous lipoid pneumonia. Indian J Pediatr. 2016;83(8):787-791. doi:10.1007/ s12098-015-2028-4

3. Albus C, Waller C, Fritzsche K, et al. Significance of psychosocial factors in cardiology: update 2018: position paper of the German cardiac society. Clin Res Cardiol. 2019;108(11):1175-1196. doi:10. 1007/s00392-019-01488-w

4. Teo I, Krishnan A, Lee GL. Psychosocial interventions for advanced cancer patients: a systematic review. Psychooncology. 2019;28 (7):1394-1407. doi:10.1002/pon.5103

5. Sias SM, Daltro PA, Marchiori E, et al. Clinic and radiological improvement of lipoid pneumonia with multiple bronchoalveolar lavages. Pediatr Pulmonol. 2009;44(4):309. doi:10.1002/ppul.20918

6. Rennick JE, Johnston CC, Dougherty G, Platt R, Ritchie JA. Children's psychological responses after critical illness and exposure to invasive technology. J Dev Behav Pediatr. 2002;23(3):133-144. doi:10.1097/00004703-200206000-00002

7. Al-Yateem N, Brenner M, Shorrab AA, Docherty C. Play distraction versus pharmacological treatment to reduce anxiety levels in children undergoing day surgery: a randomized controlled non-inferiority trial. Child Care Health Dev. 2016;42(4):572-581. doi:10.1111/cch.12343

8. Crellin DJ, Harrison D, Santamaria N, Huque H, Babl FE. The psychometric properties of the FLACC scale used to assess procedural pain. J Pain. 2018;19(8):862-872. doi:10.1016/j.jpain.2018. 02.013

9. Hoffman LR, Yen EH, Kanne JP, Effmann EL, Gibson RL, Van Niel CW. Lipoid pneumonia due to Mexican folk remedies: cultural barriers to diagnosis. Arch Pediatr Adolesc Med. 2005;159 (11):1043-1048. doi:10.1001/archpedi.159.11.1043

10. Marangu D, Pillay K, Banderker E, Gray D, Vanker A, Zampoli M. Exogenous lipoid pneumonia: an important cause of interstitial lung disease in infants. Respirol Case Rep. 2018;6(7):e00356. doi:10.10 $02 / \mathrm{rcr} 2.356$

11. Marangu D, Gray D, Vanker A, Zampoli M. Exogenous lipoid pneumonia in children: a systematic review. Paediatr Respir Rev. 2020;33:45-51.

12. Rodriguez CM, Clough V, Gowda AS, Tucker MC. Multimethod assessment of children's distress during noninvasive outpatient medical procedures: child and parent attitudes and factors. $J$ Pediatr Psychol. 2012;37(5):557-566. doi:10.1093/jpepsy/jss005

13. McMurtry MC, Chambers CT, McGrath PJ, Asp E. When "don't worry" communicates fear: children's perceptions of parental reassurance and distraction during a painful medical procedure. Pain. 2010;150(1):52-58. doi:10.1016/j.pain.2010.02.021

14. Claar RL, Walker LS, Smith CA. The influence of appraisals in understanding children's experiences with medical procedures. J Pediatr Psychol. 2002;27(7):553-563. doi:10.1093/jpepsy/27.7.553

15. Stremler R, Haddad S, Pullenayegum E, Parshuram C. Psychological outcomes in parents of critically ill hospitalized children. $J$ Pediatr Nurs. 2017;34:36-43. doi:10.1016/j.pedn.2017.01.012
International Journal of General Medicine

\section{Publish your work in this journal}

The International Journal of General Medicine is an international, peer-reviewed open-access journal that focuses on general and internal medicine, pathogenesis, epidemiology, diagnosis, monitoring and treatment protocols. The journal is characterized by the rapid reporting of reviews, original research and clinical studies across all disease areas. The manuscript management system is completely online and includes a very quick and fair peer-review system, which is all easy to use. Visit http://www.dovepress.com/ testimonials.php to read real quotes from published authors. 